\title{
Protée
}

\section{Présentation : le Groupe $\mu$ entre rhétorique et sémiotique. Archéologie et perspectives}

\section{Sémir Badir et Maria Giulia Dondero}

Volume 38, numéro 1, printemps 2010

Le Groupe $\mu$ entre rhétorique et sémiotique

URI : https://id.erudit.org/iderudit/039697ar

DOI : https://doi.org/10.7202/039697ar

Aller au sommaire du numéro

Éditeur(s)

Département des arts et lettres - Université du Québec à Chicoutimi

ISSN

0300-3523 (imprimé)

1708-2307 (numérique)

Découvrir la revue

Citer ce document

Badir, S. \& Dondero, M. (2010). Présentation : le Groupe $\mu$ entre rhétorique et sémiotique. Archéologie et perspectives. Protée, 38(1), 5-8.

https://doi.org/10.7202/039697ar d'utilisation que vous pouvez consulter en ligne.

https://apropos.erudit.org/fr/usagers/politique-dutilisation/ 
PRÉSENTATION

\title{
Le Groupe $\mu$ entre rhétorique et sémiotique Archéologie et perspectives
}

\author{
SÉMIR BADIR ET MARIA GIULIA DONDERO
} majeurs, Rhétorique générale (1970), Rhétorique de la poésie (1977) et Traité du signe visuel. Pour une rhétorique de l'image (1992). Ces trois œuvres ont chacune contribué à la renommée internationale du Groupe, l'instaurant comme un acteur de premier plan dans le débat d'idées en rhétorique et en sémiotique. C'est à rendre compte de ce débat et de la place prépondérante que les travaux du Groupe $\mu$ y occupent que se consacre le présent dossier.

Entre rhétorique et sémiotique

Les jalons que constituent les ouvrages du Groupe $\mu$ montrent au sein du projet théorique une inflexion qu'il est intéressant de remettre en question. À l'articulation d'un modèle, celui d'une rhétorique fondamentale ${ }^{1}$, vers des domaines d'applications, vient se surajouter une seconde articulation, qui apparaît aussi nécessaire que la première, celle de ce modèle avec le programme théorique de la sémiotique. D'où la possibilité d'un double, sinon triple, questionnement sur chacune de ces articulations ainsi que sur leur interaction.

Le premier examen, le plus attendu, concerne donc l'application d'un modèle théorique général à des domaines empiriques particuliers. II ne fait pas de doute que ce que la modélisation présentée dans Rhétorique générale doit à la linguistique et à son objet naturel (les langues) demande à être adapté lorsque sont envisagées d'autres régions de la semiosis, telles que l'image publicitaire, la peinture, le cinéma ou la musique. Un terme aussi central dans ce modèle que celui de figure est ainsi configuré différemment selon les substances d'expression auxquelles on l'assigne. En retour, sa conception théorique est réévaluée, élargie et enrichie à chaque application nouvelle.

Quelles sont les causes précises de ces réévaluations? Comment ont-elles infléchi le projet même d'une rhétorique générale? Telles sont les questions qu'abordent les contributions de Jean-François Bordron et de Nicolas Meeùs et JeanPierre Bartoli. L'article de Bordron, intitulé «Rhétorique et économie des images », s'interroge en fait sur la rhétorique en tant que méréologie et plus précisément en tant que «gestuelle opérant sur des totalités et des parties». Cette approche permet de comprendre comment demeure toujours sous-jacente à des sémiotiques particulières la même question de composition et de formes de liaison plus ou moins grammaticales d'unités et de traits - que ceux-ci se présentent sous forme d'arguments verbaux ou de couleurs. En suivant ce point de vue, Bordron indique la manière dont l'analyse des images peut apporter des réponses à des questions sémiotiques de portée générale sur la perception, l'iconicité et la prédication. L'article de Meeùs et Bartoli, "Sémiotique et rhétorique musicales: la Fantaisie en ré mineur de Mozart», se consacre à la question du rapport entre la rhétorique générale et une rhétorique particulière, en l'occurrence musicale. En considérant la musique comme un langage doté d'une morphologie et d'une syntaxe, les auteurs profitent de la théorie psychologique de la Gestalt, à la base de la sémiotique visuelle du Groupe $\mu$, pour démontrer comment la musique joue sur des isotopies et des allotopies rythmiques construisant des effets pathémiques particuliers analysables. 
Le deuxième examen que suscite le parcours de pensée du Groupe $\mu$ met en lumière le lien existant entre la rhétorique et la sémiotique. Ce lien n'a cessé en effet de se fortifier, en multipliant les fils qui le composent. Une rhétorique, pour être fondamentale, doit atteindre un niveau d'abstraction proche de celui réalisé par les théoriciens fondateurs de la sémiotique, Peirce et Hjelmslev notamment. Et une rhétorique ne peut se dire véritablement générale qu'à la condition d'envisager des domaines d'application aussi variés que le sont ceux couverts par la semiosis. En outre, confronté à l'application de son modèle aux domaines de l'image, le Groupe $\mu$ s'est assigné la tâche de contribuer au projet d'instauration et au développement de la sémiotique visuelle. Et, bien qu'un certain nombre de travaux sémiotiques précèdent les siens en la matière, un large consensus reconnaît aujourd'hui au Traité du signe visue/ un statut fondateur. Il faut être attentif cependant à l'orientation donnée par le Groupe $\mu$ à ces fondements théoriques de sémiotique visuelle: ceux-ci devaient, initialement, servir de base pour une rhétorique de l'image. Comment les deux projets ont-ils été appariés I'un à l'autre? Comment se sont-ils mutuellement influencés? À quelles conditions peuvent-ils être conjoints? Les questions épistémologiques occasionnées par la rencontre de la rhétorique et de la sémiotique ne manquent pas d'intérêt et débordent d'ailleurs largement le cadre strict de cette rencontre ${ }^{2}$. Ce dossier est aussi l'occasion d'un examen de la réflexion théorique sur la rhétorique et sur la spécificité de son objet général (le rhétorique). Ainsi, comment la théorie ressaisit-elle le concept d'image, tel qu'il continue de concurrencer celui de trope ou de métaphore, dès lors que la rhétorique aborde le domaine du visuel? Quelle unité profonde ou quelle confusion enfouie se loge dans ces deux acceptions, rhétorique et empirique, de l'image?

La contribution de Maria Giulia Dondero, «Rhétorique des figures visuelles et argumentation par images dans le discours scientifique», examine la relation entre sémiotique et rhétorique visuelles au sein des travaux du Groupe $\mu$ pour la mettre ensuite en parallèle avec la manière dont cette relation est également instaurée dans le cadre de la théorie post-greimassienne de l'énonciation en acte. Ce faisant, I'auteure cherche à mettre en évidence les différentes manières de concevoir la spécificité de la rhétorique visuelle par rapport à des projets disciplinaires globaux. La comparaison lui permet d'envisager le dépassement de l'étude du trope en tant que figure locale allotopique pour rendre compte des enjeux argumentatifs produits par la disposition et l'enchaînement d'images et textes verbaux à l'échelle du discours, incluant une rhétorique des gestes productifs et des tons discursifs.

En outre, la prise en compte du discours scientifique comme champ d'application de ces hypothèses amène Dondero à s'interroger sur la créativité ${ }^{3}$ conceptuelle des figures discursives, telle l'exemplification figurale. Cette question est aussi au centre de l'étude de Michele Prandi, "L'interaction métaphorique: une grandeur algébrique». Ce dernier, en reparcourant la tradition théorique de la métaphore, vise à isoler le phénomène qui est à la source de toute métaphore, mais seulement de la métaphore (le transfert qui déclenche une interaction entre concepts provenant de milieux d'appartenance étrangers), afin de concevoir un processus unitaire qui puisse inclure toutes les issues historiquement attestées. En distinguant les métaphores cohérentes (accessibles par la pensée indépendamment de leur expression linguistique) des métaphores conflictuelles (où, au contraire, la pensée est infléchie par l'expression linguistique), l'auteur montre que ces dernières permettent une adaptation de deux environnements conceptuels en conflit - dans le cas du solde positif, une véritable projection dépasse le besoin de résolution et de cohérence linguistique interne en dilatant I’horizon conceptuel de son efficace créative.

Enfin, la troisième approche, qui permettra de saisir la particularité historique et épistémologique de l'œuvre du Groupe $\mu$, consiste à observer de quelles autres formes de pensée la rencontre avec la sémiotique aura éloigné la rhétorique. Au moins trois disciplines méritent d'être mentionnées à cet égard. Premièrement, la linguistique, dite structurale, a sans aucun doute inspiré non seulement le modèle présenté dans Rhétorique générale, mais aussi son titre (par celui des Essais de linguistique générale de Roman Jakobson) et son projet même. Pourtant, la linguistique n'a pu que céder le pas devant la sémiotique quand le champ d'application du modèle s'est étendu aux domaines de l'image. À son tour, 
la poétique aurait pu, au temps de Rhétorique de la poésie et alors que la sémiotique se tournait elle-même largement vers des questions relevant du champ des études littéraires, constituer l'interlocuteur privilégié de la rhétorique du Groupe $\mu$. La poétique est d'ailleurs ouverte, non moins que la rhétorique, à des considérations qui concernent toutes les formes artistiques. Enfin, la troisième discipline, la stylistique, partage avec la rhétorique bien des questionnements, notamment autour des notions d'écart, d'énonciation et d'effet. Force est de constater toutefois que ce n'est ni avec les

stylisticiens, ni avec les poéticiens, que le Groupe $\mu$ a eu les dialogues les plus nourris. Quels enjeux ces éloignements ont-ils servis? Ou encore, si le terme d'enjeu paraît faire trop peu de cas des circonstances, quels effets ont-ils eus sur l'évolution de la rhétorique du Groupe $\mu$ ? Voilà une troisième série d'interrogations qui, tout en suivant le parcours d'une œuvre, cerne dans le même temps celui d'une discipline au contact de son voisinage épistémique.

La question de la concurrence et du voisinage entre rhétorique et stylistique est abordée dans l'article de Madeleine Frédéric, "Les travaux du Groupe $\mu$ : amers pour la stylistique? », où l'auteure montre, au fil d'analyses d'œuvres poétiques, la portée heuristique de la rhétorique du Groupe $\mu$. Cette rhétorique permet non seulement de s'éloigner de la tendance esthético-évaluative de la stylistique, mais aussi de redéfinir cette dernière à travers les notions de forme de l'expression et forme du contenu empruntées à la sémiotique de Hjelmslev. Il devient ainsi possible d'étudier les relations entre isotopies du contenu et les corrélations entre isotopies du contenu et isotopies de l'expression - l'approche de ces dernières ayant été renouvelée grâce à la notion de tabularité mise en place par le Groupe $\mu$. C'est d'ailleurs par la tabularité que Frédéric décrit les relations intersémiotiques entre poésie et image, démontrant ainsi que la prise en compte de la sémiotique à l'intérieur du champ de la stylistique permet aussi de comparer les fonctionnements des rhétoriques particulières entre elles.

\section{Archéologie et perspectives}

Les recherches qui portent sur le projet théorique du Groupe $\mu$ ont deux versants indissociables: historique et critique (ou épistémologique). Elles évaluent un parcours, mais prospectent aussi pour l'avenir. Dans tous les cas, elles exigent qu'on se penche sur la situation du projet du Groupe $\mu$ dans des contextes correspondant à diverses échelles épistémiques.

Dans le cercle le plus étroit, celui de la genèse d'un tel projet, il importe de savoir comment celui-ci s'est inscrit dans le courant d'idées qui avait le vent en poupe, à savoir le structuralisme.

En élargissant le cercle aux dimensions de la discipline, la rhétorique du Groupe $\mu$ est souvent comparée, et bientôt opposée, à la rhétorique instaurée, une dizaine d'années auparavant, par Chaïm Perelman. Les deux projets, développés tous deux en Belgique, sont également désignés comme «nouvelle rhétorique» et vont évoluer en parallèle, sans se rejoindre, tout en lorgnant sur leurs avancées respectives.

L'article de Michel Meyer, "Pour une théorie générale des figures», témoigne d'une attention réciproque: si Klinkenberg est revenu ailleurs sur la nécessité de concevoir la rhétorique en tant que négociation entre des individus ayant des compétences et des intérêts différents, Meyer rappelle ici les quatre opérations fondamentales de la rhétorique, mises en évidence par le Groupe $\mu$, tout en les transformant en dispositifs nécessaires pour comprendre différents niveaux de figurativité (des figures de son aux figures de pensée en passant par les figures de mots et les tropes). L'élargissement philosophique de la perspective rhétorique et de sa technique, tel qu'il est opéré par Meyer, est dû, premièrement, à une mise en question de la possibilité de distinguer le sens littéral du sens figuré et, deuxièmement, à la reformulation de cette ancienne distinction qui oscille entre questions et réponses dans le cadre d'une problématologie. Le trope serait ainsi une réponse problématique à une question non explicitement formulée, ce qui amène l'auteur à voir dans les figures et dans l'enchaînement de réponses plus ou moins équivoques, plus ou moins dynamisantes, le déploiement de la connaissance elle-même. 
Dans le champ plus large encore des sciences du langage, dont l'élaboration est concomitante du parcours du Groupe $\mu$, les aspirations inévitablement généralistes des différents théoriciens du champ vont les conduire à porter leurs réflexions sur l'objet rhétorique et sur sa place dans une configuration plus large. C'est en particulier le cas en sémiotique, dans sa mouvance post-greimassienne et contemporaine, où le rhétorique est l'objet d'une élaboration théorique conséquente, intégrant l'action d'une figure dans le cadre d'une analyse des pratiques discursives (littéraires et artistiques, mais aussi scientifiques ou politiques), révélant par là même la dimension énonciative de cette figure et ses effets de sens, y compris les effets pathémiques, sur le récepteur.

La question de la relation entre figure et énonciation, si elle est abordée dans la contribution de Dondero, devient centrale dans I'article de Marc Bonhomme, «La rhétorique des figures: entre formalisme et énonciation ». La rhétorique des figures ayant oscillé entre ces deux pôles difficiles à harmoniser, l'étude trace une histoire de ce rapport de manière à faire ressortir comment le Groupe $\mu$ a repris les catégories de Quintilien en les dynamisant et en les hiérarchisant à la lumière de la linguistique moderne. L'auteur voit dans Rhétorique générale, et dans les travaux successifs de Jean-Marie Klinkenberg, un apport majeur dans la constitution d'une rhétorique intégrée qui concilie le donné sémiotique des figures et leur actualisation en discours: les figures deviennent ainsi des structures discursives modelées par leur prise en charge énonciative et par les intentions des sujets communicants.

Enfin, le cercle le plus large rejoint le plus étroit en ce qu'il met en avant la possibilité de changements paradigmatiques dans l'approche théorique du rhétorique. De structuraliste, la pensée du Groupe $\mu$ a pu se faire de plus en plus pragmatiste pour intégrer en définitive une problématique proprement cognitiviste. Ce chemin est reparcouru par Sémir Badir dans l'article qui ouvre le dossier. Ses «Éléments pour une biographie du Groupe $\mu$ » posent les jalons d'un récit où les données personnelles sont intégrées aux données qui concernent les disciplines et les effets de concurrences se jouant entre celles-ci. Aussi l'auteur décrit-il les choix disciplinaires et épistémologiques du Groupe en constituant une dynamique de figure/fond avec les mouvements et les prises de position d'un monde "en fibrillation », faisant ressortir la relation d'adaptation et d'action du Groupe à ce monde.

En somme, à chaque échelle permettant de situer le projet de la rhétorique du Groupe $\mu$, il aura paru important de prendre en considération l'évolution de parcours épistémiques concomitants au sien. C'est à cette condition que les contributions du présent dossier se font les témoins des conditions et enjeux qui viennent d'être brièvement esquissés. Elles instaurent avec la pensée du Groupe $\mu$ une dialogique, tantôt critique, tantôt participative, mettant en relief ses acquis comme ses singularités.

\footnotetext{
1. Tel est le titre de la première partie - la plus conséquente et la plus commentée - de Rhétorique générale. Ce n'est que dans la deuxième partie de cet ouvrage, où sont abordés les domaines de l'interlocution et de la narration, que le Groupe $\mu$ donne un début de réalisation à son projet de rhétorique générale.

2. Sur cette question, nous renvoyons le lecteur à M. G. Dondero et G. Sonesson (dir.), «Le Groupe $\mu$. Quarante ans de rhétorique Trente trois de sémiotique visuelle», Nouveaux Actes Sémiotiques. En ligne: http://revues.unilim.fr/nas/sommaire.php?id=3246 (page consultée le 9 mars 2010).

3. Au sujet de la créativité rhétorique au sein du sémiotique, voir aussi J.-M. Klinkenberg, "La rhétorique dans le sémiotique: la composante créative du système», et les autres contributions contenues dans S. Badir et J.-M. Klinkenberg (dir.), Figures de la figure. Rhétorique et sémiotique générale, Limoges, Pulim, 2008.
} 